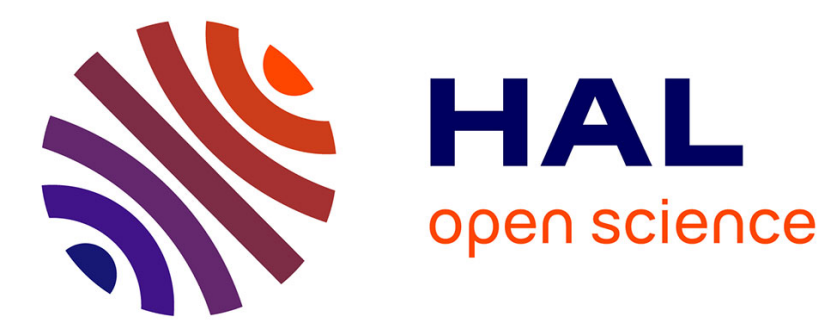

\title{
High order Lagrangian velocity statistics in turbulence
}

Haitao Xu, Mickaël Bourgoin, Nicholas T. Ouellette, Eberhard Bodenschatz

\section{To cite this version:}

Haitao Xu, Mickaël Bourgoin, Nicholas T. Ouellette, Eberhard Bodenschatz. High order Lagrangian velocity statistics in turbulence. Physical Review Letters, 2006, 96 (2), pp.024503. 10.1103/PhysRevLett.96.024503 . hal-00492364

\section{HAL Id: hal-00492364 https://hal.science/hal-00492364}

Submitted on 15 Jun 2010

HAL is a multi-disciplinary open access archive for the deposit and dissemination of scientific research documents, whether they are published or not. The documents may come from teaching and research institutions in France or abroad, or from public or private research centers.
L'archive ouverte pluridisciplinaire HAL, est destinée au dépôt et à la diffusion de documents scientifiques de niveau recherche, publiés ou non, émanant des établissements d'enseignement et de recherche français ou étrangers, des laboratoires publics ou privés. 


\title{
High Order Lagrangian Velocity Statistics in Turbulence
}

\author{
Haitao Xu, ${ }^{1,2}$ Mickäl Bourgoin, ${ }^{3}$ Nicholas T. Ouellette, ${ }^{1}$ and Eberhard Bodenschatz ${ }^{1,2, *}$ \\ (International Collaboration for Turbulence Research) \\ ${ }^{1}$ Laboratory of Atomic and Solid State Physics, Cornell University, Ithaca, New York 14853, USA \\ ${ }^{2}$ Max Planck Institute for Dynamics and Self-Organization, 37077 Göttingen, Germany \\ ${ }^{3}$ Laboratoire des Écoulements Géophysiques et Industriels-C.N.R.S., BP 53-38041, Grenoble Cedex 9, France
}

(Received 14 September 2005; published 19 January 2006)

\begin{abstract}
We report measurements of the Lagrangian velocity structure functions of orders 1 through 10 in a high Reynolds number (Taylor microscale Reynolds numbers of up to $R_{\lambda}=815$ ) turbulence experiment. Passive tracer particles are tracked optically in three dimensions and in time, and velocities are calculated from the particle tracks. The structure function anomalous scaling exponents are measured both directly and using extended self-similarity and are found to be more intermittent than their Eulerian counterparts. Classical Kolmogorov inertial range scaling is also found for all structure function orders at times that trend downward as the order increases. The temporal shift of this classical scaling behavior is observed to saturate as the structure function order increases at times shorter than the Kolmogorov time scale.
\end{abstract}

PACS numbers: 47.27.Gs, 02.50.-r, 47.27.Jv, 89.75.Da

Since its publication in 1941, Kolmogorov's scaling theory [1], often abbreviated simply as K41, has been the dominant phenomenological model of fluid turbulence. The great utility of the K41 model lies in its prediction of universal scaling laws for all manner of turbulence statistics. Of such statistics, the moments of spatial velocity differences $\delta u(r)=u(x+r)-u(x)$ are of particular importance, since the Kolmogorov 4/5 law, one of the only results in turbulence theory exactly derivable from the Navier-Stokes equations, concerns the third moment of $\delta u(r)$. K41 predicts that the moments $\left\langle|\delta u(r)|^{p}\right\rangle$ should scale as $r_{p}^{\zeta_{p}^{E}}$ with $\zeta_{p}^{E}=p / 3$ in the inertial range of turbulence where the only relevant flow parameter is the rate of energy dissipation per unit mass $\epsilon$. Closer studies, however, have shown that the K41 scaling predictions are not obeyed, especially for high-order moments. Instead, the $\zeta_{p}^{E}$ increase nonlinearly and slower than $p / 3$ [2-4]. This anomalous scaling is usually attributed to the phenomenon of intermittency that destroys the perfect self-similarity underlying the K41 model [3].

Almost all of the observations of anomalous scaling have been made for Eulerian quantities like $\left\langle|\delta u(r)|^{p}\right\rangle$, measured with probes fixed relative to some laboratory reference frame. K41 also applies, however, to Lagrangian quantities measured along the trajectories of individual fluid elements. Historically, Lagrangian measurements have been very difficult to perform. For this reason, anomalous scaling and intermittency have not been fully investigated from the Lagrangian viewpoint. Given that the Lagrangian approach is more natural for many problems in turbulence [5], a more thorough understanding of Lagrangian intermittency is needed. In addition, experimental measurements of Lagrangian anomalous scaling provide data to test models of turbulence [6-8].
In this Letter, we study the high-order moments of the Lagrangian velocity difference $\delta u(\tau)=u(t+\tau)-u(t)$ experimentally in a high Reynolds number turbulent flow using 3D optical Lagrangian particle tracking. According to $\mathrm{K} 41$, these moments, also known as the Lagrangian structure functions, should scale as

$$
D_{p}^{L}(\tau)=\left\langle|\delta u(\tau)|^{p}\right\rangle \sim(\epsilon \tau)^{\zeta_{p}^{L}}
$$

in the inertial range, with $\zeta_{p}^{L}=p / 2$. As mentioned above, there have been few experimental studies of the deviation of the $\zeta_{p}^{L}$ from the K41 prediction, due both to the inherent difficulty in making Lagrangian measurements and to the high degree of temporal resolution required to measure the high-order structure functions. Mordant et al. [9] measured Lagrangian velocity statistics in an acoustical particle tracking system in a flow similar to ours, and found that the Lagrangian structure functions showed more intermittency than their Eulerian counterparts. Because of the requirements of their acoustical system, however, their tracer particles were much larger than the Kolmogorov length scale $\eta$ and they recorded data at a rate slower than the Kolmogorov time scale $\tau_{\eta}$. The measured particle tracks may therefore have smeared out the smallest length and time scales of the flow. This problem of resolution is easily solved in simulation. Biferale et al. [7,8] investigated the Lagrangian structure functions in a direct numerical simulation (DNS) of the Navier-Stokes equations in the context of the multifractal formalism. As with many simulations, however, their results are limited by their low Reynolds number. This is also true of the simulation of Mazzitelli and Lohse [10], who studied Lagrangian statistics both for fluid elements and for bubbles. 
Our experimental facility consists of a closed cylindrical chamber containing $0.1 \mathrm{~m}^{3}$ of water. We generate turbulence via the counter-rotation of two baffled disks driven by $1 \mathrm{~kW} \mathrm{dc}$ motors, and the temperature of the water is controlled to within $0.1{ }^{\circ} \mathrm{C}$. A more detailed description of the apparatus was given in a previous report [11]. In order to measure Lagrangian statistics, we seed the flow with transparent polystyrene microspheres with a diameter of $25 \mu \mathrm{m}$, which is smaller than or comparable to the Kolmogorov length scale $\eta$ for all three Reynolds numbers tested. These microspheres have a density 1.06 times that of water, and have been shown to act as passive tracers in our flow [11]. The microspheres are illuminated with one $\sim 90 \mathrm{~W}$ and one $\sim 60 \mathrm{~W}$ pulsed Nd:YAG laser, and their motion is tracked using Lagrangian particle tracking algorithms [12] in a subvolume of $(2.5 \mathrm{~cm})^{3}$ in the center of the tank where the effects of the mean flow are negligible. In order to achieve the high time resolution necessary to resolve high-order Lagrangian structure functions, we image the tracers using high speed digital cameras. As sketched in Fig. 1, we use three Phantom v7.1 CMOS cameras from Vision Research, Inc., which can record images at up to 27000 frames per second at a resolution of $256 \times 256$ pixels, arranged in a single plane with an angular separation of $45^{\circ}$ in the forward scattering direction from both lasers. Once the raw particle tracks have been obtained, they are processed to obtain Lagrangian velocities by convolution with a Gaussian smoothing and differentiating kernel [13]. In this Letter, we report only the

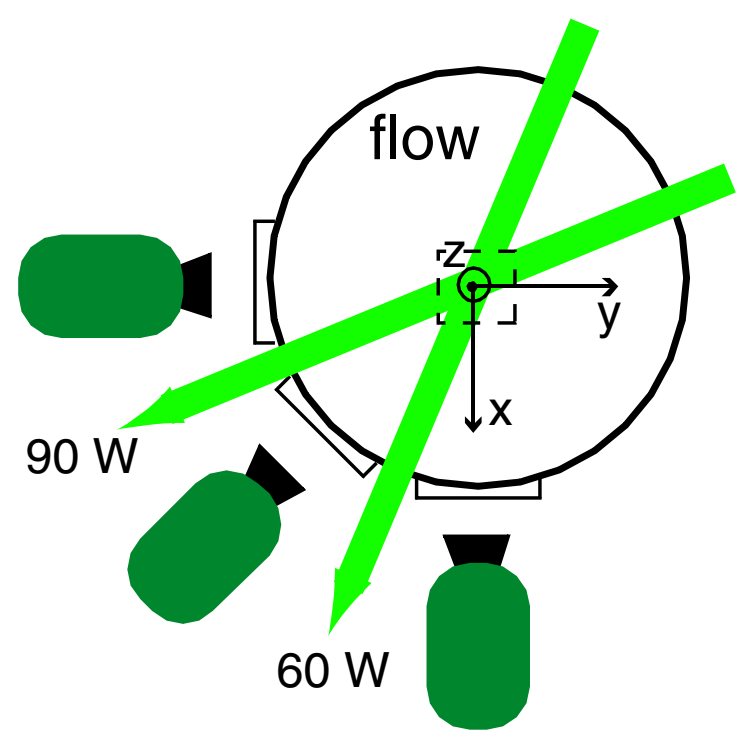

FIG. 1 (color online). Top-down view of the experiment. The dashed box represents the $(2.5 \mathrm{~cm})^{3}$ measurement volume. The tracer particles were illuminated with one $\sim 90 \mathrm{~W}$ and one $\sim 60 \mathrm{~W}$ pulsed $\mathrm{Nd}: Y A G$ laser aligned so that the cameras were in the forward scattering direction from both beams. The three cameras were arranged in a plane with an angular separation of $45^{\circ}$. The disks rotated about the $z$ axis. structure functions measured from the radial velocity components.

In recent years, the extended self-similarity ansatz introduced by Benzi et al. [14] has become a widely used tool for investigating the anomalous scaling of the Eulerian $\zeta_{p}^{E}$. This technique is based on the Kolmogorov 4/5 law mentioned above. Kolmogorov was able to show rigorously from the Navier-Stokes equations that $\zeta_{3}^{E}=1$ [15]. Therefore, $\left\langle|\delta u(r)|^{p}\right\rangle \sim r^{\zeta_{p}^{E}} \sim\left\langle|\delta u(r)|^{3}\right\rangle^{\zeta_{p}^{E}}$ exactly. Plotting the structure functions of different orders against each other tends to produce cleaner scaling ranges since imperfections in the scaling behavior in the near dissipation range seem to be correlated among structure functions of different order [3]; this fact may also point to the existence of new universal functions with the same scaling exponents in the near dissipation range [16]. Regardless, extended selfsimilarity (ESS) has been shown to produce very welldetermined values of the $\zeta_{p}^{E}$.

Because of its great utility in determining the scaling exponents of the Eulerian structure functions, researchers have extended the ESS ansatz to the Lagrangian structure functions [7-9], using the fact that K41 scaling gives $\zeta_{2}^{L}=$ 1. While this result has not been proved rigorously from the Navier-Stokes equations, the fact that the K41 scaling law for the second order structure function is linear in the energy dissipation rate $\epsilon$ suggests that intermittency effects should not change the value of $\zeta_{2}^{L}$ [17]. In Fig. 2, we plot the Lagrangian structure functions of orders 1 through 10 as measured in our experiment at a Reynolds number of $R_{\lambda}=815$ using ESS.

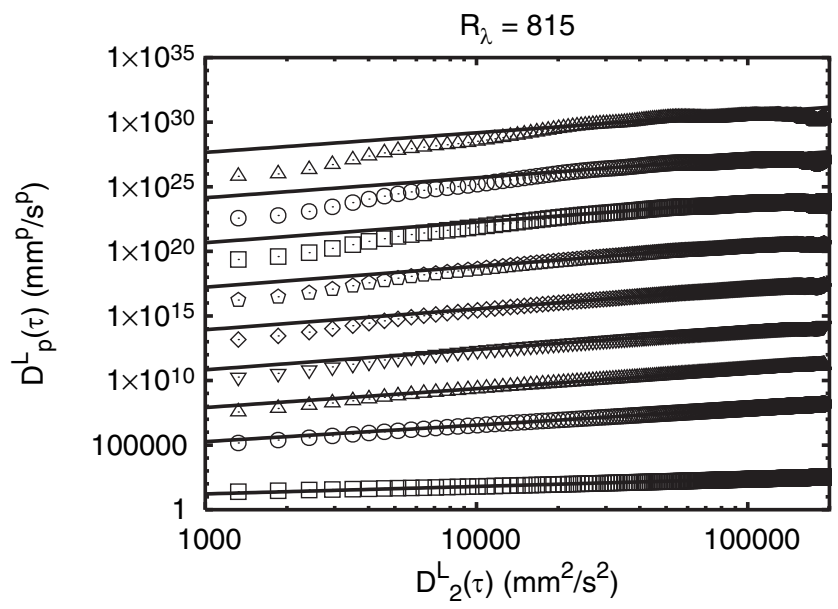

FIG. 2. ESS plot of the high-order Lagrangian structure functions at $R_{\lambda}=815$. From top to bottom, the symbols correspond to our measurements of the tenth order through first order structure function, with second order omitted. The straight lines are fits to the data to extract the relative scaling exponents. The lines were fit only to values of $D_{2}^{L}(\tau)$ corresponding to times between $3 \tau_{\eta}$ and $6 \tau_{\eta}$, where $D_{2}^{L}(\tau)$ displayed a K41 scaling range with $\zeta_{2}^{L} \approx 1$. 


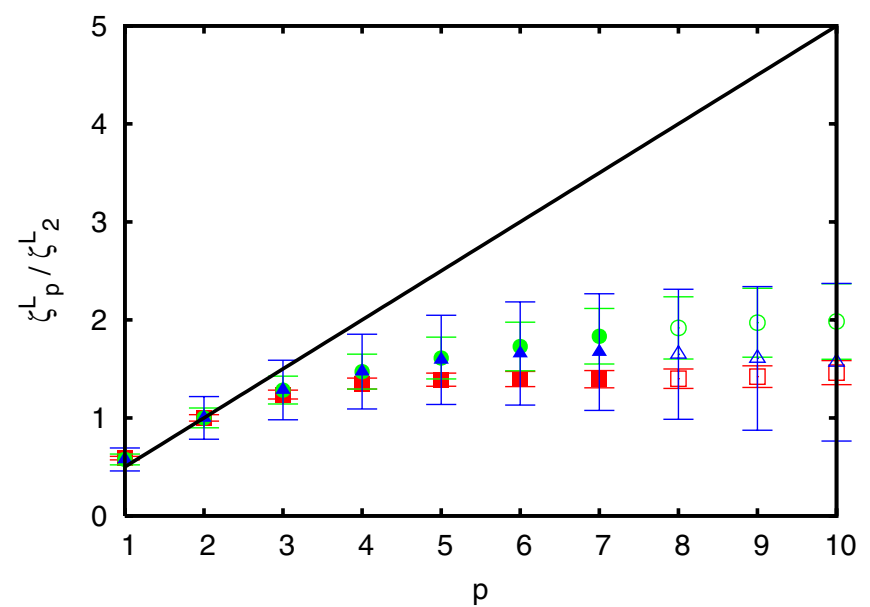

FIG. 3 (color online). Anomalous scaling of the structure function relative scaling exponents $\zeta_{p}^{L} / \zeta_{2}^{L}$ measured using ESS as a function of order. The solid line shows the K41 prediction for the scaling exponents, with $\zeta_{2}^{L}=1$. Different symbols denote different Reynolds numbers: the red ( $\square$ ) are for $R_{\lambda}=200$, the green $(\mathbf{O})$ are for $R_{\lambda}=690$, and the blue $(\boldsymbol{\Delta})$ are for $R_{\lambda}=$ 815. Strong departure from the K41 prediction is clear for all Reynolds numbers investigated. Equivalent results are found without using ESS (not shown). Moments of orders higher than 7 are not as well converged statistically as the lower-order moments, as suggested by their larger error bars. These highorder moments are plotted with open symbols.

Because of the lack of an exact equation for any of the $\zeta_{p}^{L}$ similar to the Kolmogorov 4/5 law, ESS can only be strictly used to measure relative scaling exponents in the Lagrangian case. Figure 3 shows our measurements of the relative exponents $\zeta_{p}^{L} / \zeta_{2}^{L}$ computed using ESS. Since $\zeta_{2}^{L}$ should be close to unity, this ratio should be close to the true value of $\zeta_{p}^{L}$. In order to find the relative exponent as close as possible to $\zeta_{p}^{L}$, we have fit straight lines to the ESS curves only between $D_{2}^{L}\left(3 \tau_{\eta}\right)$ and $D_{2}^{L}\left(6 \tau_{\eta}\right)$ for $R_{\lambda}=690$ and 815 and $D_{2}^{L}\left(2 \tau_{\eta}\right)$ and $D_{2}^{L}\left(4.5 \tau_{\eta}\right)$ for $R_{\lambda}=200$, where a very limited K41 scaling range for the second order structure function is evident. In analogy with the usual Eulerian definition [3], we take this range to be the Lagrangian inertial range. These fits are shown in Fig. 2. The values of the relative scaling exponents are shown in Table I, and are compared with the K41 predictions in
Fig. 3. It is clear that there is significant deviation from the $\mathrm{K} 41$ prediction, and that this deviation is stronger than in the Eulerian case. This behavior has also been observed by Mordant et al. [9]. Additionally, since the ESS ansatz has not been fully justified theoretically, we have also measured the scaling exponents without using ESS; these absolute scaling exponents are nearly identical to the ESS values.

The scaling exponents shown in Fig. 3 and Table I are similar to those measured by Mordant et al. [9], who measured up to sixth order. Both our results and those of Mordant et al. [7,8], however, are significantly lower than the findings of Biferale et al. [7,8] who made predictions based on a simple extension of the successful Eulerian multifractal model to the Lagrangian case. Biferale et al. $[7,8]$ fit power laws to their structure functions for times between $10 \tau_{\eta}$ and $50 \tau_{\eta}$. These times were long enough, however, that they fell outside of the Lagrangian inertial range reported by Biferale et al. [8] for their simulations. Fitting their structure functions for times shorter than $10 \tau_{\eta}$ led them to find scaling exponents near 2 [8], in much better agreement with our results. They attribute this clustering of exponents near 2 to intense small scale vortical motion characterized by intense acceleration [8].

We have seen from Figs. 2 and 3 that the scaling properties of the high-order Lagrangian structure functions are anomalous when measured for time ranges where the second order structure function shows a K41 scaling region, which we have assumed corresponds to the Lagrangian inertial range. If we scale the structure functions by the K41 prediction, however, a different picture emerges. As shown in Fig. 4, the higher order structure functions do indeed show plateaus when compensated by the K41 predictions, albeit at shorter times than for the low order structure functions. The open circles in Fig. 4 show the centers of the K41 scaling ranges, which occur at times we denote by $t_{\mathrm{K} 41}$.

Figure 4 suggests that the value of $t_{\mathrm{K} 41}$ decreases and saturates at a value smaller than $\tau_{\eta}$ as the structure function order increases. We have observed this effect for all three Reynolds numbers investigated, as shown in Fig. 5. While $t_{\mathrm{K} 41}$ is smaller for the low order structure functions at $R_{\lambda}=$ 200 than at the higher Reynolds numbers, the $R_{\lambda}=200$ results collapse with the higher Reynolds number data at

TABLE I. Values of the relative scaling exponents measured in our experiment using ESS. The ESS curves were fit only in the range of times where the second order structure function displayed a K41 scaling range with exponent $\zeta_{2}^{L} \approx 1$. For comparison, we included the values measured from the DNS of Biferale et al. [8] and the experiment of Mordant et al. [9]

\begin{tabular}{|c|c|c|c|c|c|c|c|c|c|c|}
\hline & $R_{\lambda}$ & $\zeta_{1}^{L} / \zeta_{2}^{L}$ & $\zeta_{3}^{L} / \zeta_{2}^{L}$ & $\zeta_{4}^{L} / \zeta_{2}^{L}$ & $\zeta_{5}^{L} / \zeta_{2}^{L}$ & $\zeta_{6}^{L} / \zeta_{2}^{L}$ & $\zeta_{7}^{L} / \zeta_{2}^{L}$ & $\zeta_{8}^{L} / \zeta_{2}^{L}$ & $\zeta_{9}^{L} / \zeta_{2}^{L}$ & $\zeta_{10}^{L} / \zeta_{2}^{L}$ \\
\hline & 200 & $0.59 \pm 0.02$ & $1.24 \pm 0.03$ & $1.35 \pm 0.04$ & $1.39 \pm 0.07$ & $1.40 \pm 0.08$ & $1.39 \pm 0.09$ & $1.40 \pm 0.10$ & $1.42 \pm 0.11$ & $1.46 \pm 0.12$ \\
\hline & 690 & $0.58 \pm 0.05$ & $1.28 \pm 0.14$ & $1.47 \pm 0.18$ & $1.61 \pm 0.21$ & $1.73 \pm 0.25$ & $1.83 \pm 0.28$ & $1.92 \pm 0.32$ & $1.97 \pm 0.35$ & $1.98 \pm 0.38$ \\
\hline & 815 & $0.58 \pm 0.12$ & $1.28 \pm 0.30$ & $1.47 \pm 0.38$ & $1.59 \pm 0.46$ & $1.66 \pm 0.53$ & $1.67 \pm 0.60$ & $1.65 \pm 0.66$ & $1.61 \pm 0.73$ & $1.57 \pm 0.80$ \\
\hline Ref. [8] & 284 & & & $1.7 \pm 0.05$ & $2.0 \pm 0.05$ & $2.2 \pm 0.07$ & & & & \\
\hline Ref. [9] & 740 & $0.56 \pm 0.01$ & $1.34 \pm 0.02$ & $1.56 \pm 0.06$ & $1.73 \pm 0.1$ & $1.8 \pm 0.2$ & & & & \\
\hline
\end{tabular}




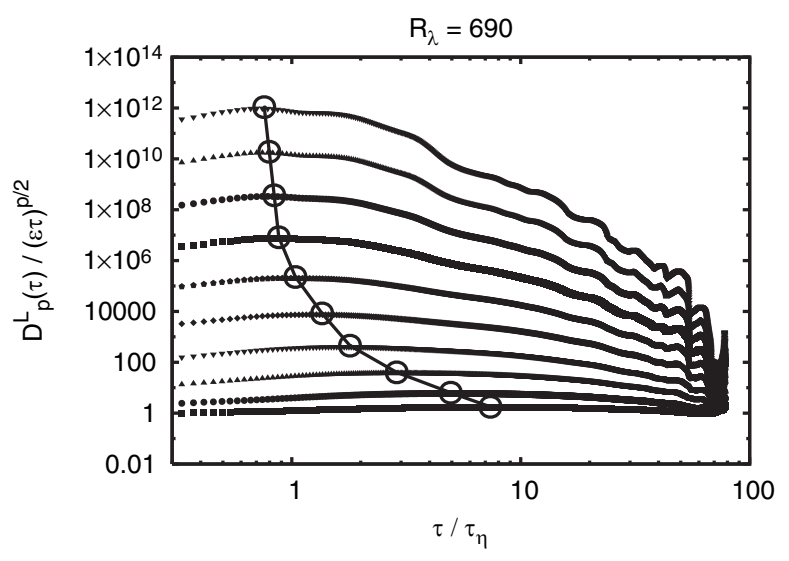

FIG. 4. High-order Lagrangian structure functions at $R_{\lambda}=$ 690 compensated by the K41 predictions. The order of the structure function increases from 1 to 10 from the bottom curve to the top curve. A K41 scaling region is seen at all orders, but this plateau shifts to shorter times as the order of the structure function increases, as shown by the open circles.

high order. For all three Reynolds numbers, $t_{K 41}$ saturates at high order at times smaller than $\tau_{\eta}$. This shift of the K41 inertial range towards the dissipation range may also be found in the Eulerian case [18].

In summary, we have used our high speed optical particle tracking system to measure the scaling properties of the Lagrangian velocity structure functions up to order 10 in a high Reynolds number turbulence experiment. Using the extended self-similarity ansatz, we have measured the anomalous scaling exponents $\zeta_{p}^{L} / \zeta_{2}^{L}$ for time ranges corresponding to the K41 scaling range of the second order structure function. We have found that these scaling exponents show stronger intermittency corrections than their

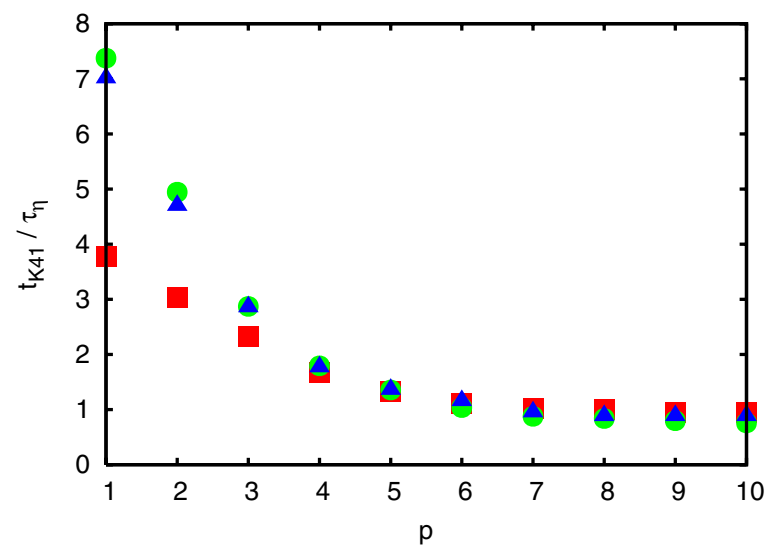

FIG. 5 (color online). Shift of the effective inertial range as a function of structure function order $p$. Different symbols denote different Reynolds numbers: the red $(\boldsymbol{\square})$ are for $R_{\lambda}=200$, the green $(\boldsymbol{O})$ are for $R_{\lambda}=690$, and the blue $(\mathbf{\Lambda})$ are for $R_{\lambda}=815$. For higher-order structure functions, the K41 inertial range scaling region appears at the same multiple of $\tau_{\eta}$ for all Reynolds numbers investigated.
Eulerian counterparts. In addition, we have shown that for all structure function orders we find a K41 scaling range for times of the order of the Kolmogorov time $\tau_{\eta}$. This K41 scaling region is characterized by a time $t_{\mathrm{K} 41}$ that varies with structure function order but appears to saturate to times smaller than $\tau_{\eta}$ for high orders for all three Reynolds numbers measured. While the finite size of our tracer particles might affect the highest-order statistics presented in this Letter [19], we hope that our results will spur further understanding of the Lagrangian picture of turbulence.

This work was supported by the NSF under Grants No. PHY-9988755 and No. PHY-0216406 and by the Max Planck Society.

*Electronic address: eberhard.bodenschatz@ds.mpg.de

[1] A. N. Kolmogorov, Dokl. Akad. Nauk SSSR 30, 301 (1941) [Proc. R. Soc. A 434, 9 (1991)].

[2] F. Anselmet, Y. Gagne, E. J. Hopfinger, and R. A. Antonia, J. Fluid Mech. 140, 63 (1984).

[3] U. Frisch, Turbulence: The Legacy of A. N. Kolmogorov (Cambridge University Press, Cambridge, England, 1995).

[4] S. Y. Chen, B. Dhruva, S. Kurien, K. R. Sreenivasan, and M. A. Taylor, J. Fluid Mech. 533, 183 (2005).

[5] P. K. Yeung, Annu. Rev. Fluid Mech. 34, 115 (2002).

[6] L. Chevillard, S. G. Roux, E. Lévêque, N. Mordant, J.-F. Pinton, and A. Arneodo, Phys. Rev. Lett. 91, 214502 (2003).

[7] L. Biferale, G. Boffetta, A. Celani, B. J. Devenish, A. Lanotte, and F. Toschi, Phys. Rev. Lett. 93, 064502 (2004).

[8] L. Biferale, G. Boffetta, A. Celani, A. Lanotte, and F. Toschi, Phys. Fluids 17, 021701 (2005).

[9] N. Mordant, P. Metz, O. Michel, and J.-F. Pinton, Phys. Rev. Lett. 87, 214501 (2001).

[10] I. M. Mazzitelli and D. Lohse, New J. Phys. 6, 203 (2004).

[11] G. A. Voth, A. La Porta, A. M. Crawford, J. Alexander, and E. Bodenschatz, J. Fluid Mech. 469, 121 (2002).

[12] N. T. Ouellette, H. Xu, and E. Bodenschatz, Exp. Fluids (to be published).

[13] N. Mordant, A. M. Crawford, and E. Bodenschatz, Physica D (Amsterdam) 193, 245 (2004).

[14] R. Benzi, S. Ciliberto, R. Tripiccione, C. Baudet, F. Massaioli, and S. Succi, Phys. Rev. E 48, R29 (1993).

[15] A. N. Kolmogorov, Dokl. Akad. Nauk SSSR 32, 16 (1941) [Proc. R. Soc. A 434, 15 (1991)].

[16] K. R. Sreenivasan and A. Bershadskii, Pramana-J. Phys. 64, 315 (2005).

[17] G. Boffetta, F. De Lillo, and S. Musacchio, Phys. Rev. E 66, 066307 (2002).

[18] K. R. Sreenivasan, LANL Center for Nonlinear Studies Workshop on Multi-Scale Interactions in Turbulent Flows, 2005 (unpublished).

[19] V. Yakhot, J. Fluid Mech. 495, 135 (2003). 